\title{
Study on the development of Traditional Chinese Medicine Hospitals in Northwest China under the background of the Belt and Road Initiative
}

\author{
Guifang Liu ${ }^{1}$, Lina Shuai ${ }^{1}$, li Chen ${ }^{1}$, Changmin Tang ${ }^{1}$, Yuanxi Xiang ${ }^{1 *}$ \\ ${ }^{1}$ School of Management, Hubei University of Chinese Medicine, 430065, Wuhan, Hubei, China
}

\begin{abstract}
To understand the development status of Traditional Chinese Medicine(TCM) hospitals in Northwest China under the background of the Belt and Road initiative(B\&R), this study analyses the health resource index of TCM hospitals in Northwest China, and finds that the development of TCM hospitals in Qinghai and Ningxia is relatively slow, the recognition of ethnomedical services is relatively low among the residents. Regional linkage could be used to co-ordinately facilitate the development of TCM hospitals in Northwest China. B\&R could be taken advantage of to promote the high-quality development of local ethnic medicine. Cultivating composite TCM talents, promoting poverty alleviation with TCM services, advocating TCM culture could all be utilized to build the People-to-people bonds along the new Silk Road.
\end{abstract}

\section{Introduction}

At a time when the global economic development is weak and when the development of various countries is divided, China has proposed a win-win cooperation plan, that is, the initiative of jointly building the B\&R. In this initiative, China demonstrates its demeanor, as a responsible great country, shares development dividends with countries along the route with an inclusive and open mind, and promotes the coordinated development of regions along the route. The five Northwest provinces and autonomous regions of Shaanxi, Gansu, Qinghai, Ningxia and Xinjiang are located at the strategic frontiers of the Silk Road Economic Belt. Due to their unique geographical location and historical and cultural advantages, these regions have become the stronghold of China's opening up to the outside world. TCM had played an important role and become an important pillar of the development of B\&R. TCM hospital is a medical centre where TCM and other ethnomedical healthcare services were provided, and has ushered in a new opportunity for fast growth under the national strategy of promoting TCM development[1].

According to the 2019 National TCM Statistical Digest, traditional medicine hospitals in Shaanxi, Gansu and Ningxia were mainly composed of TCM(of Han-Chinese) hospitals, whereas traditional medicine hospitals in Qinghai were mainly composed of ethnomedical(of minority ethnic groups) hospitals. Xinjiang had almost equally amount of hospital in both types. TCM resources in Northwest China are rich in variety and has its only ethnic characteristics. The number of institutions, beds and health technicians of TCM hospitals in five provinces and autonomous regions in Northwest China have increased by a certain amount from 2015 to 2019 . As of the end of 2019 , in Shaanxi, the number of TCM hospitals has increased by 21 , the number of beds in TCM hospitals has increased by 7,928 , and the number of health technicians in TCM hospitals has increased by 10,158; in Gansu, the number of TCM hospitals has increased by 69 , the number of beds has increased by 9,854 , and the number of health technicians increased by 10,768; in Qinghai, the number of TCM hospitals increased by 11, the number of beds increased by 821 , and the number of health technicians increased by 1,529; in Ningxia, the total number of TCM hospitals increased by 9 , the number of beds increased by 1,496 , and the number of health technicians has increased by 2,033 ; in Xinjiang, the number of TCM hospitals has increased by 13 , the number of beds has increased by 5607 , and the number of health technicians has increased by 5,519. The scale of local TCM hospitals has been expanding, and the overall development trend is good as shown in Table 1 .

The service status of TCM hospitals was measured by analysing the number of inpatients and outpatients[2]. As shown in Table 2, the overall number of patients in the five regions are on the increase. Compared with 2015, as of the end of 2019, the number of visits and discharges in traditional Chinese medicine hospitals in Shaanxi increased by 3674 thousand and 323 thousand respectively; the number of visits and discharges in traditional Chinese medicine hospitals in Gansu increased by 3783 thousand and 353 thousands respectively; the number of visits and discharges in TCM hospitals in Qinghai increased by 470 thousand and 22 thousand, respectively; TCM hospitals in Ningxia increased by 701 thousand and 54 thousand in visits and discharges; TCM in Xinjiang The number of hospital visits and discharges increased by 1,854 thousand and 214 thousand, respectively. It suggests that the public

\footnotetext{
${ }^{*}$ Corresponding author: xiangyx@hbtcm.edu.cn
} 
recognition of TCM serves is increasing under the environment of great government support and "prevention-oriented" health policies

Table1. TCM hospitals number of 5 provinces in Northwest China

\begin{tabular}{|c|c|c|c|c|c|c|c|c|c|}
\hline \multirow{2}{*}{ Province } & \multicolumn{3}{|c|}{ Number of institutions } & \multicolumn{3}{|c|}{ Number of beds } & \multicolumn{3}{|c|}{ Number of health technicians } \\
\hline & 2015 & 2019 & Growth & 2015 & 2019 & Growth & 2015 & 2019 & Growth \\
\hline Shaanxi & 163 & 184 & 21 & 27271 & 35199 & 7928 & 27122 & 37280 & 10158 \\
\hline Gansu & 103 & 172 & 69 & 22031 & 31885 & 9854 & 12610 & 23378 & 10768 \\
\hline Qinghai & 47 & 58 & 11 & 5444 & 6265 & 821 & 3549 & 5078 & 1529 \\
\hline Ningxia & 24 & 33 & 9 & 4192 & 5688 & 1496 & 3353 & 5386 & 2033 \\
\hline Xinjiang & 108 & 121 & 13 & 17784 & 23391 & 5607 & 12705 & 18224 & 5519 \\
\hline
\end{tabular}

Table2. Outpatients and inpatient number of TCM hospitals of 5 provinces in Northwest China

\begin{tabular}{cccccccc}
\hline & \multicolumn{2}{c}{ Outpatient number (in thousand) } & & \multicolumn{3}{c}{ Inpatient number (in thousand) } \\
\cline { 2 - 3 } \cline { 6 - 8 } Province & 2015 & 2019 & Growth & & 2015 & 2019 & Growth \\
\hline Shaanxi & 11523 & 15197 & 3674 & & 768 & 1091 & 323 \\
Gansu & 8947 & 12730 & 3783 & & 604 & 957 & 353 \\
Qinghai & 1989 & 2459 & 470 & & 112 & 134 & 22 \\
Ningxia & 3019 & 3720 & 701 & & 113 & 166 & 54 \\
Xinjiang & 6261 & 8115 & 1854 & & 527 & 741 & 214 \\
\hline
\end{tabular}

\section{Problems in the development of TCM hospitals in Northwest China under the Background B\&R initiative}

\subsection{The development of TCM hospitals in Qinghai and Ningxia is relatively slow}

As of the end of 2019, the province with relatively rich resources of Chinese medicine hospitals in Northwest China is Shaanxi, followed by Gansu and Xinjiang, while Qinghai and Ningxia have relatively few Chinese medicine hospitals. There are a total of 184 TCM hospitals in Shaanxi, with a total of 35,199 beds in TCM hospitals, and a total of 37,280 health technicians in TCM hospitals; a total of 172 TCM hospitals in Gansu with a total of 31,885 beds and 23,378 technicians in TCM hospitals; there are 121 TCM hospitals in Xinjiang with 23,391 beds in TCM hospitals and 18,224 health technicians in TCM hospitals. There are only 58 TCM hospitals in Qinghai with 6,265 beds and 5,078 hygienic technicians. Ningxia has 33 TCM hospitals and TCM hospitals have 5,688 beds and there are 5386 health technicians in such hospitals. From the perspective of the service development of traditional Chinese medicine hospitals in various regions, the total number of visits of traditional Chinese medicine hospitals in Shaanxi is 15,197 thousand, and the total number of discharged people from traditional Chinese medicine hospitals is 1091 thousand; the total number of visits of traditional Chinese medicine hospitals in Gansu is 12,730 thousand, and the number of people discharged from TCM hospitals totaled 957 thousand; the total number of patients treated in Xinjiang TCM hospitals totaled 8115 thousand, and the number of discharged TCM hospitals totaled 741 thousand. Qinghai and Ningxia Chinese medicine hospitals have relatively low number of visits and discharges. Qinghai's Chinese medicine hospitals have a total of 24,59 thousand visits, and Chinese medicine hospitals have discharged a total of 134 thousand; Ningxia's TCM hospitals had a total of 37,20 thousand patients for diagnosis and treatment, and TCM hospitals had discharged a total of 166 thousand people._Compared with other 5 provinces in the northwest region, Qinghai and Ningxia are at the bottom of the list in terms of the number of institutions, beds, health technicians, and number of inpatients and outpatients, which shows a relative lack of TCM resources. The relatively slow growth rate of TCM institutions and beds in Qinghai and Ningxia, as shown in Table 1 and 2, implies that the TCM resources were relatively scarce and development was relatively slow as well.

\subsection{Relatively low public recognition of ethnomedical services}

Ethnic medicine is the essence of medicine formed by ethnic minority people in the process of fighting diseases, and is an "important member" of traditional medicine in my country. On the road to TCM revival, no minority 
ethnomedicine should fall behind. The scale of traditional medicine hospitals in the northwest is expanding in recent years, but the number of outpatients and inpatients in ethnomedical hospitals were much lower than that in TCM hospitals. For example, traditional medicine hospitals in Qinghai are mainly composed of ethnomedical hospitals, and there were 14 Chinese medicine hospitals, 7 integrated Chinese and Western medicine hospitals and 37 ethnomedical hospitals in the year of 2019. However, the number of outpatients was 1,572,000 and the number of outpatients was 82,410 in Chinese medicine hospitals, while the number of outpatients was just 737,000 and number of outpatients was 48,439 in ethnomedical hospitals, which suggests that the service capacity of TCM hospitals exceeds far beyond that of the ethnic medicine hospitals.

\subsection{The shortage of composite TCM talents hinders the internationalization of TCM}

Health technicians are the core force in hospitals development, and the shortage of TCM talents has always been a persistent barrier in the development of TCM in China. Judging from the national rankings of the "Number of Chinese Medicine Practicing Physicians (including TCM Practicing Assistants)" in the provinces and autonomous regions of Northwest China in 2019, Shaanxi ranks 18th, Gansu ranks 9th and Qinghai ranks 7th, Ningxia ranks 16th, Xinjiang ranks 22nd, and the relatively small population of Northwest China shows that the number of local TCM physicians is not optimistic. The personnel training method of TCM is unique, mostly in "teacher-apprentice" way. The training cycle is long, and there has always been a shortage of TCM talents. Under the background of B\&R initiative, TCM service are being widely used in countries all over the world[3], and the requirements and demands for TCM talents have increased. Domestic TCM hospitals need composite TCM talents to support B\&R projects such as building international departments and carrying out medical service assistance overseas. Only in this way can TCM hospitals really be internationalized, and TCM culture be promoted to the world.

\section{Suggestions for promoting TCM in Northwest China under the background of $B \& R$}

\subsection{Promote the integration of TCM industry in the Northwest China through regional linkage}

Under the B\&R strategy, which advocates freedom of trade, the five northwestern provinces and autonomous regions should seize the opportunity to carry out in-depth cooperation and exchange with neighboring provinces in order to enhance the market circulation in Northwest China. Regional economic development should be promoted through regional linkage and collaboration[4]. Northwest regions have their own advantages in TCM, and
Shaanxi and Gansu province have relatively abundant resources in TCM hospitals, while Qinghai, Ningxia and Xinjiang provinces are rich ethnic medicine resources according to data analysis and relevant policy reports. Thus, Northwest China should promote the integration of TCM industry according to their respective advantages, accelerate regional TCM development under the background of B\&R initiative, and promoting the quality TCM services internationally. Specifically, Shaanxi's rich resources of TCM hospitals can be fully utilized to carry out regional TCM technology transfer activities to help the development of TCM hospitals in Qinghai and Ningxia; Gansu will continue to promote the construction of a strong TCM province and strengthen the characteristics of TCM in the northwest region, cultivate of medicinal materials, share experience in poverty alleviation with traditional Chinese medicine for the provinces and autonomous regions in Northwest China, leading the Northwest Region to carry out poverty alleviation with traditional Chinese medicine; Qinghai and Ningxia give full play to the advantages of local ethnic medical culture, learn from the development path of traditional Chinese medicine, and integrate with neighboring countries along the B\&R Medical demand promotes the revitalization of ethnic medical culture; Xinjiang uses the construction of the "Silk Road Economic Core Area Medical Service Center" to do a good job related to opening to the west, turning the local area into a window for the western region to open to the west, and assuming regional quality Chinese medicine services go out to be a heavy burden. The Northwest region can give full play to the potential of local TCM hospitals and enhance the service capabilities of local TCM services in order to make better use of the advantages of the Silk Road strategic position and promote the revitalization and development of local high-quality traditional medicine.

\subsection{Take advantage of the B\&R initiative to promote the high-quality development of traditional ethnic medicine}

China has always been paying attention to the protection and exploration of traditional medicine, and keeping a good momentum of development in TCM. However, the development of minority ethnic medicine is in a disadvantaged position. People-to-people bonds is the basis for promoting reginal cooperation under the background of B\&R initiative, and ethnic medicine has cultural ties with neighboring countries across time and space, catering the practical needs of people along the Belt and the Road regions[5]. Northwest regions should take the leading role of ethnomedical hospitals and integrate quality ethnic medicine resources. Ethnomedical experts should learn from the successful experience of internationalization of Chinese medicine, seize the opportunity to actively carry out health service provision in neighboring countries so as to strengthen the exchange and cooperation mutually, and finally promote the highquality development of local ethnic medicine. 


\subsection{Cultivate composite TCM talents, promote poverty alleviation with TCM services, and advocate TCM culture}

Poverty eradication is the common vision of people all around the world, and TCM has played an important role in the overall victory of poverty alleviation in China. Traditional medicine has the advantage of being "Simple, Effective and inexpensive"[6]. It is reported that the TCM services have promoted the construction of Malaysia's health system, and the people's demand for Chinese medicine services has continued to grow[7].The advantages of TCM could help to improve the regional essential health network and reduce the burden of the poor. On the road of joint construction of the B\&R initiative, spreading the experience of TCM poverty alleviation to the people of the world and promoting global poverty reduction will not only benefit the international development of TCM, but also demonstrate the responsibility of a major country. With the help of the B\&R of traditional Chinese medicine to bring traditional Chinese medicine medical technical assistance to poor areas along the "Silk Road", China needs to adjust the training plan for Chinese medicine talents. TCM talents are the main providers of TCM services, in order to promote the internationalization of TCM, these medical personnel need to overcome language barriers[8]. Only through strengthening the international training of TCM talents, enhancing their professional capability and improving service awareness could they provide more friendly medical services along the $\mathrm{B} \& \mathrm{R}$ regions. In the meantime, more exchange activities of traditional medicine theory should be carried out with neighboring countries so as to spread the concept of "treating before it happens" in TCM among residents and medical professionals, relieve the burden of the local people in trouble of seeking medical services. Finally, promotion and internationalization of TCM could be achieved.

\section{Conclusions}

This article takes the B\&R Chinese medicine as the research background and selects data from the 2015-2019 China Health Statistics Yearbook and the National TCM Statistical Digest for the analysis of TCM hospitals in Northwest China. Through a horizontal comparison of the development status and development differences of TCM hospitals in the five northwestern provinces and autonomous regions, and a vertical time comparison to grasp the impact of $\mathrm{B} \& \mathrm{R}$ related policies on the development of TCM hospitals in Northwest China. The study found that there is a large gap in the development of TCM hospitals between provinces in Northwest China. The resources of TCM hospitals in Shaanxi and Gansu are relatively rich, and the resources of TCM hospitals in Qinghai and Ningxia are relatively scarce and slow in development; Comparing the data of 2015 and 2019, it is found that the policy document "Vision and proposed actions outlined on jointly building Silk Road Economic Belt and 21st-Century Maritime Silk Road" has been in good shape since the release of the policy document. The allocation of human resources and material resources has improved to a certain extent, and the service development of TCM hospitals is also relatively good. In addition, research has found that although the Northwest region is a concentrated area of ethnic minority people in China, and has rich ethnic medicine resources, the local people's recognition of ethnic medicine services is relatively low. However, the development of ethnic minority medicine in China is inherently faced with difficulties such as shortage of talents, weak competitiveness, and insufficient government support. Therefore, relevant experts and scholars should learn from the experience of Chinese medicine "going out" and use the B\&R as the engine to revitalize local traditional medicine.

The B\&R initiative is a valuable opportunity to promote TCM to people of the world. If TCM hospitals in Northwest China could keep up with the trend, make full use of local TCM resources to mutually benefit countries along the "Silk Road" and promote people-to-people bonds, it would not only help to promote the TCM culture but also benefiting the people around.

\section{References}

1. YM. Yao. Analysis of internationalization strategies of Chinese hospitals towards "one belt and one road", Jiangsu Health System Management. 29(2018)

2. W Duan, ZY Li, YM Hu, XL Wu, M Xue, W Jiang, Y Zhao. Analysis of the development trend of Chinese medicine hospital resources and services from 2003 to 2013, Chinese Journal of Health Informatics and Management, 12, 108-112 (2015)

3. XT XU, HH Tang, S Zhen. Development status and prospects of international standardization of medical devices of Traditional Chinese Medicine. PHARMACOL RES, 167(2021)

4. CY Wang, J Cheng, XH Wei. Research on regional four-position integration concept in Northwest china under the "belt and road" strategy, Journal of Xi' an University of Finance and Economics, 30, 45-51 (2017)

5. YX Wang, LL Niu, YC Bian, LJ Su, The value potential of ethnic traditional medicine in the contest of "one belt and one road", Chin J Ethnomed Ethnopharm, 27, 1-5 (2018)

6. WW Shen, JY Wang, et al. Research on the path of TCM health poverty alleviation in the context of collaborative governance, Chin Med Herald, 17, 181-184 (2020)

7. $\mathrm{SH} \mathrm{Ng,H} \mathrm{Wong,W} \mathrm{Tan} \mathrm{,J} \mathrm{Liu} \mathrm{,HY} \mathrm{Wang,} \mathrm{X} \mathrm{Lin} \mathrm{,Si}$ Goh ,B Hoo ,CY Chai. Traditional Chinese medicine in Malaysia: A brief historical overview of the institutions. Chinese Medicine and Culture,2(2019).

8. A Hinsley, E. J. Milner-Gulland, R Cooney, et al. Building sustainability into the Belt and Road Initiative's Traditional Chinese Medicine trade, NAT SUSTAIN, 3, 96-100 (2020) 